\title{
Pale Mass behind Intact Tympanic Membrane: An Otologic Puzzle to Solve
}

\author{
Gyanaranjan Nayak Manjul Muraleedharan ${ }^{1}$ \\ ${ }^{1}$ Department of Otolaryngology, Head and Neck Surgery, \\ Postgraduate Institute of Medical Education and Research, \\ Chandigarh, India \\ 2Department of Pathology, Postgraduate Institute of Medical \\ Education and Research, Chandigarh, India
}

\section{Kanika Arora ${ }^{1}$ Ramandeep Virk ${ }^{1}$ Debajyoti Chatterjee ${ }^{2}$}

Ann Otol Neurotol:2020;3:35-37

\author{
Address for correspondence Gyanaranjan Nayak, MS, DNB, \\ Department of Otolaryngology, Head and Neck Surgery, \\ Postgraduate Institute of Medical Education and Research, \\ Sector 12, Chandigarh 160012, India \\ (e-mail: gyani.nayak@gmail.com).
}
Abstract
Keywords
- middle ear mass
- paraganglioma
- nonpulsatile

Whitish pale looking mass in the middle ear space is a diagnostic challenge not only to a novice otologic surgeon but also for an experienced surgeon. The array of differentials should be known for appropriate site of origin and its true nature. We have discussed a case of pale looking tympanic paraganglioma behind an intact tympanic membrane with few differentials to consider.

\section{Introduction}

Whitish pale looking mass in middle ear cleft throws a variety of diagnosis to consider. The management of such lesion requires prior good clinical examination and appropriate radiological investigations to assess its location and extent. We have presented a case of pale looking mass in middle ear cleft with an array of differentials to consider.

\section{Case Report}

A 47-year-old male presented to the outpatient clinic with decreased hearing in left ear for the past 5 years. Hearing loss was insidious in onset and gradually progressive that he is unable to hear normal conversations with left ear. It was associated with left-sided dull aching mild ear pain and buzzing tinnitus (nonpulsatile). There was no associated otorrhea, bleeding, fever, or facial deviation. On examination, the pars tensa was intact and draped over a whitish nonpulsatile mass in the middle ear with a shallow posterosuperior retraction pocket. The high-resolution computed tomography scan of temporal bone was suggestive of soft tissue density in the left middle ear over the promontory limited to the mesotympanum with no extension into the mastoid air cells ( - Fig. 1 ). Pure tone audiometry of the left ear showed a 27/52 dB HL. After counseling and informed consent, patient was taken up for excision of mass under general anesthesia. The standard transcanal with post aural approach was done with vascular strip incision. Intraoperatively there was a soft pale looking mass involving the mesotympanum with attachment over promontory and hypotympanic air cells reaching anteriorly up to Eustachian tube opening (-Fig. 2).

The mass had minimal vascularity with insignificant bleeding on dissection and removal. The long process of incus was partially eroded with intact stapes and malleus. The lesion was excised in toto and ossiculoplasty was done with refashioned incus. The patient had uneventful recovery. The histopathological examination revealed a monomorphic tumor arranged in clusters and trabeculae separated by thin fibrous septae. The individual tumor cells had round to oval nuclei, few of which were eccentrically placed, powdery chromatin, and moderate eosinophilic cytoplasm (-Fig. 3 ). Areas of hemorrhage were also identified. No mitosis, necrosis, or atypia was seen. On immunohistochemistry, it was positive for synaptophysin and CD-56 (- Fig. 4). Overall features were suggestive of tympanic paraganglioma.

\section{Discussion}

White mass behind an intact tympanic membrane is an intriguing diagnostic dilemma that could entice an otologist. What could bring only congenital cholesteatoma to the mind of an occasional ear surgeon could open a wide array of differential diagnosis to an experienced mind. An overview of the differential diagnoses of this interesting clinical presentation 


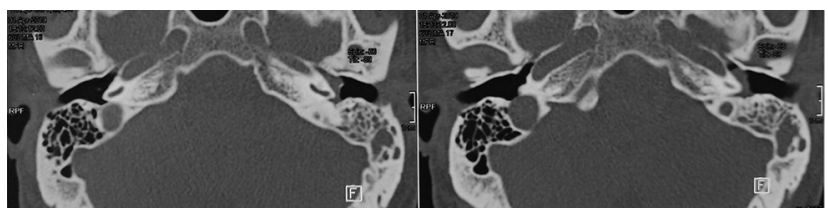

Fig. 1 HRCT temporal bone showing soft tissue density over the promontory. HRCT, high resolution computed tomography.

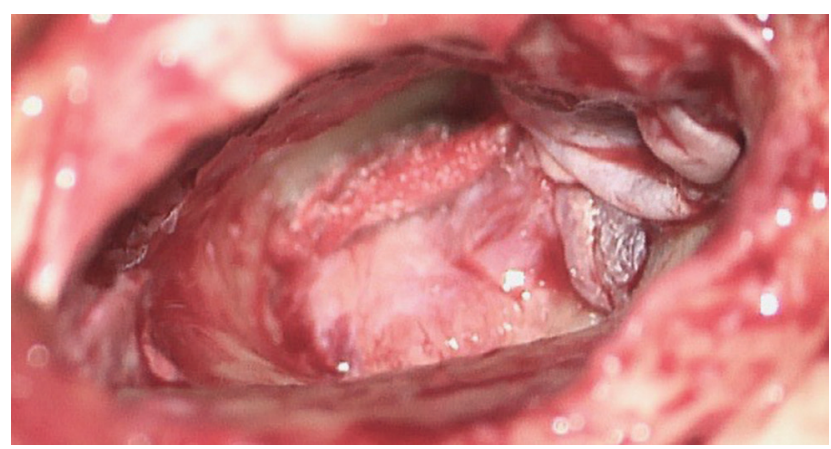

Fig. 2 Pale looking mass in the middle ear over the promontory after elevation of tympanomeatal flap.

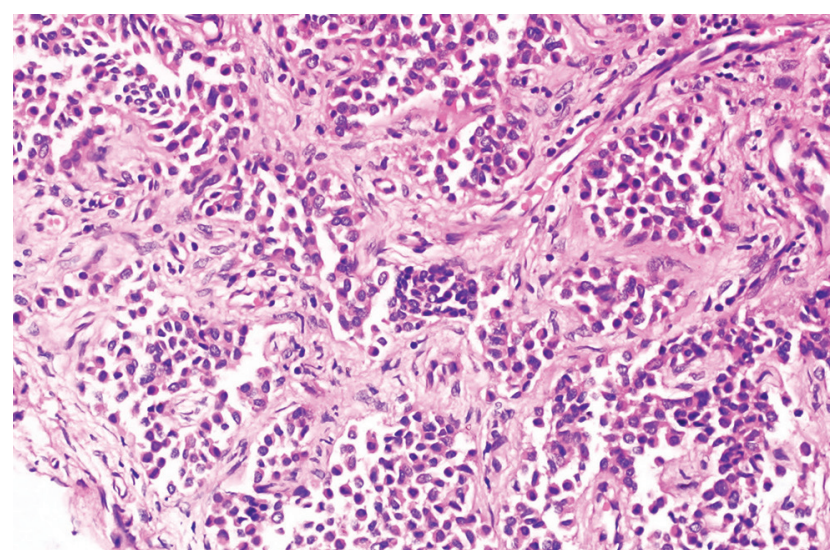

Fig. 3 Histological microphotograph with H\&E staining. H\&E staining, hematoxylin and eosin staining.

is attempted herewith. The presenting symptoms of the patients with middle ear masses are not particularly helpful in establishing the diagnosis. Owing to the location most of the patients have symptoms of aural fullness, conductive loss of hearing, tinnitus and rarely dull aching kind of pain. In spite of extensive differentials, the location and the appearance of the middle ear mass can give an idea about the histology. The classical presentation of congenital cholesteatoma is an asymptomatic white mass behind an intact tympanic membrane. The majority of the lesions were found to be present in the anterosuperior quadrant. ${ }^{1}$

Paraganglioma in this region presents with pulsatile middle ear mass. The term tympanic paraganglioma is used for masses confined to middle ear cleft with intact jugular bulb whereas jugular paraganglioma arise from the dome of

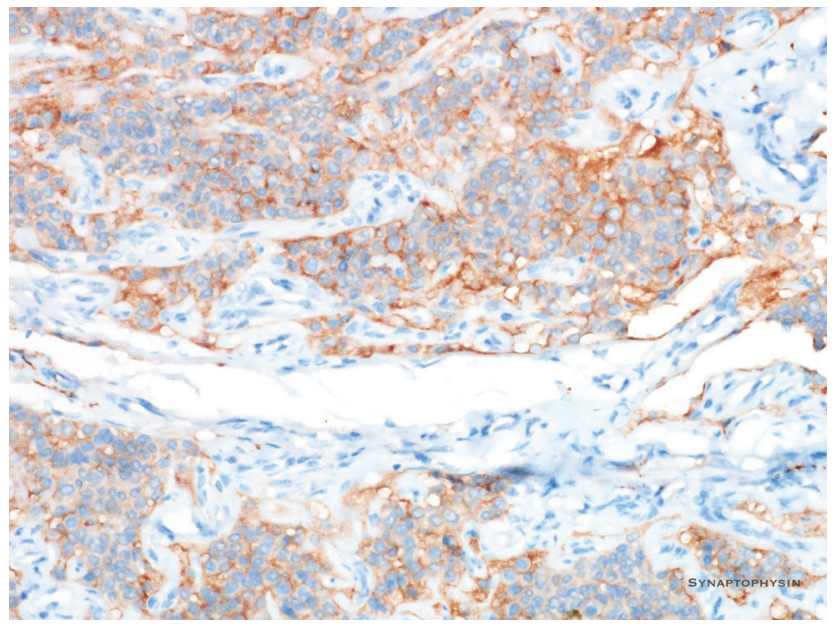

Fig. $4 \mathrm{IHC}$ with synaptophysin showing tumor cells arranged in clusters. IHC, immunohistochemistry.

jugular bulb in the hypotympanum. Due to the close proximity of tumor to hypotympanum and jugular bulb the exact site of origin is sometimes difficult to determine. ${ }^{2}$

Glomangioma or "true" glomus tumor is one of the rarest differentials for middle ear mass behind an intact tympanic membrane. Arising from the smooth muscles of glomus bodies, the clinical presentation in one of the reported cases included had whitish mass behind tympanic membrane with pulsatile tinnitus and hearing. ${ }^{3}$

A vestibular schwannoma though rare could also present in a similar way. ${ }^{4}$ Some postulate that the tumors are of intralabyrinthine in origin and extend medially toward the cerebellopontine angle and laterally into the middle ear via the round window. ${ }^{5}$ Other neurogenic tumors like facial nerve schwannoma and Jacobson's nerve schwannoma also form middle ear masses, albeit rarely. ${ }^{6}$ These masses present similarly to a glomus tumor. Even imaging findings are quite similar and virtually indistinguishable. Intraoperatively these tumors could be seen as tan-pink, firm mass with prominent superficial vasculature.

Another uncommon differential to be considered is middle ear adenoma and are same as the neuroendocrine (carcinoid) tumors. ${ }^{7}$ There are also reports of high riding jugular bulb with intact thick bone presenting as whitish mass in the mesotympanum. ${ }^{8}$

In spite of the typical location of the tumor, tympanic paraganglioma was not on the top of our list of differentials for the mass, in view of the scanty vascularity. The lesion was neither showing any pinkish or reddish hue nor pulsations on otoscopy or examination under microscope. Even though it appears that more the vascular components in the lesion, more should be the bleeding, no such correlation could be found out despite extensive search. The specimen of tympanic paraganglioma under consideration did show more than usual fibrous stroma in comparison to the vascular channels, but objective estimation of the same is very difficult, if not impossible. This aspect of vascular tumors of head and neck thus needs further studies and evaluation. 


\section{Conclusion}

A long list of differential diagnosis should be kept in mind while dealing with white pale looking mass behind an intact tympanic membrane. Intraoperative finding of less than expected bleed should not be a criterion for ruling out paraganglioma and a histopathological examination should always be obtained before making a final diagnosis.

\section{Conflict of Interest}

None declared.

\section{References}

1 Levenson MJ, Parisier SC, Chute P, Wenig S, Juarbe C. A review of twenty congenital cholesteatomas of the middle ear in children. Otolaryngol Head Neck Surg 1986;94(5):560-567

2 Jackson CG. Glomus tympanicum and glomus jugulare tumors. Otolaryngol Clin North Am 2001;34(5):941-970
3 Choi JH. Glomangioma ("true" glomus tumor) presenting as a white middle ear mass behind an intact tympanic membrane. Otol Neurotol 2012;33(6):e51-e52

4 Ebmeyer J, Gehl HB, Upile T, Sudhoff HH. Vestibular schwannoma presenting as a white middle ear mass behind an intact tympanic membrane. Otol Neurotol 2011;32(5):e32-e33

5 Yamada S, Aiba T, Takada K, Takemori S, Kumakawa K. A rare case of acoustic neuroma extending from the cerebellopontine angle to the external auditory canal. J Clin Neurosci 1998;5(1):94-97

6 Lin KF, Turk AT, Kim AH. Jacobson's nerve schwannoma presenting as a middle ear mass causing pulsatile tinnitus: a case report. Otol Neurotol 2017;38(9):e354-e356

7 Torske KR, Thompson LD. Adenoma versus carcinoid tumor of the middle ear: a study of 48 cases and review of the literature. Mod Pathol 2002;15(5):543-555

8 Lee DH, Kim J. White mass in the middle ear cavity behind an intact tympanic membrane. Otolaryngol Head Neck Surg 2013;148(6):1054-1055 